\title{
Optimized Monte Carlo Method for glasses
}

\author{
L. A. Fernández ${ }^{1,3, *}$ V. Martín-Mayor ${ }^{1,3, \dagger}$ and P. Verrocchio ${ }^{2,3,4 \ddagger}$ \\ ${ }^{1}$ Dep. de Física Teórica I, U. Complutense, 28040 Madrid, Spain. \\ ${ }^{2}$ Dip. di Fisica, U. di Trento, 38050 Povo, Trento, Italy. \\ ${ }^{3}$ Instituto de Biocomputación y Física de Sistemas Complejos (BIFI). Zaragoza, Spain. \\ ${ }^{4}$ Research center Soft INFM-CNR c/o U. di Trento 38050 Povo, Trento, Italy.
}

September 22, 2018

\begin{abstract}
A new Monte Carlo algorithm is introduced for the simulation of supercooled liquids and glass formers, and tested in two model glasses. The algorithm is shown to thermalize well below the Mode Coupling temperature and to outperform other optimized Monte Carlo methods. Using the algorithm, we obtain finite size effects in the specific heat. This effect points to the existence of a large correlation length measurable in equal time correlation functions.
\end{abstract}

\footnotetext{
${ }^{*}$ laf@lattice.fis.ucm.es, ${ }^{\dagger}$ victor@lattice.fis.ucm.es, ${ }^{\ddagger}$ paolo.verrocchio@unitn.it
} 


\section{Introduction}

The lack of structural or thermodynamic changes at the glass transition [1] is a major problem for its investigation. The only standard feature, as compared with second-order phase transitions [2, is the dramatic dynamical slowing down at the critical temperature. A fairly standard mechanism for slow dynamics in an homogeneous system at finite temperature is the divergence of a correlation length (critical slowing down [2]). Slowness arises from the need of configurational changes to propagate over increasingly large regions (the critical origin of the Mode Coupling singularity $[3]$ has been recently recognized [4]). It has been recently proposed [5] to study this growing lengthscale in glassformers through the finite size behaviour of small systems [2]. Note that experiments in films and nanopores [6, 7 . show that the glass transition changes in samples with one or more dimensions of nanometric scale. In particular, the specific heat is most sensitive to the size of the confining pore when temperatures are close to the glass transition $[\underline{8}$.

Numerical simulations are an important tool for the study of the glass transition. Their worse drawback is the shortness of the times that may be simulated in today computers (roughly speaking, microseconds). As a consequence, the computer model goes out of equilibrium by the Mode-Coupling temperature, $T_{\mathrm{mc}}$, rather than the actual glass temperature, $T_{\mathrm{c}}$. To approach $T_{\mathrm{c}}$, one may resort to optimized Monte Carlo (MC) methods 9, 10, 5], namely methods implementing unphysical dynamical rules that strongly reduce the equilibration times. When thermalization is achieved, optimized MC allow to study equilibrium mean values and their temperature (or pressure) derivatives, although the purely dynamic features of these methods are interesting on their own right [5].

Here, we give the first full description of the local swap algorithm [5]. We compare the performance of the local swap dynamics with the standard MC and with the microcanonical algorithm [10. We conclude that local swap yield equilibrium data at temperatures where the microcanonical algorithm no longer thermalizes. Finally, we address the fishy issue of estimating the specific heat in a metastable liquid state. We give here details on the strategy followed in [5], where tiny but clearly measurable finite-size effects were observed in the specific heat.

\section{Models and observables}

We consider two similar models of fragile glass formers, namely binary mixtures of soft spheres. The first model, extensively studied in Ref. [5], is a 50\% mixture of particles interacting through the pair potential $V_{\alpha \beta}(r)=\epsilon\left[\left(\sigma_{\alpha}+\sigma_{\beta}\right) / r\right]^{12}+C_{\alpha \beta}$, where $\alpha, \beta=A, B$, with a cutoff at $r_{\mathrm{c}}=\sqrt{3} \sigma_{0}$. The choice $\sigma_{A}=1.2 \sigma_{B}$ hampers crystallization, as compared with the $\sigma_{A}=\sigma_{B}$ model. We impose $\left(2 \sigma_{A}\right)^{3}+$ $2\left(\sigma_{A}+\sigma_{B}\right)^{3}+\left(2 \sigma_{B}\right)^{3}=4 \sigma_{0}^{3}$ where $\sigma_{0}$ is the unit length. Constants $C_{\alpha \beta}$ are chosen to ensure continuity at $r_{\mathrm{c}}$. The simulations are at constant volume, with particle density fixed to $\sigma_{0}^{-3}$ and temperatures in the range $\left[0.897 T_{\mathrm{mc}}, 10.792 T_{\mathrm{mc}}\right]$. We use periodic boundary conditions on a box of size $L$ in systems with $N=512,1024,2048$ and 4096 particles. For argon parameters, $\sigma_{0}=3.4 \AA, \epsilon / k_{B}=120 \mathrm{~K}$ and $T_{\mathrm{mc}}=26.4 \mathrm{~K}$.

In the second model [11, 10] the choice $\sigma_{B}=1.4 \sigma_{A}$ is made. Naming $x=r /\left(\sigma_{\alpha}+\sigma_{\beta}\right)$, the pair potential in units $\epsilon=1$ is $V(x)=x^{-12}+x-13 / 12^{12 / 13}$ if $x<12^{1 / 13}$ and zero otherwise (thus, the cut-off distance depends on the type of interacting species). We study density $1.08 \sigma_{0}^{-3}$, as in Refs. [11, 10].

Since the potential energy per particle, $e$ shows the slowest excitations [5] 11, we shall focus here only in this observable, the internal energy being $\frac{3}{2} k_{\mathrm{B}} T+\langle e\rangle$ (for other quantities, see Ref. [5]). The constant-volume specific heat, $C_{v}$, is:

$$
e=\frac{1}{2 N} \sum_{j, k \neq j} V\left(\vec{r}_{k}-\vec{r}_{j}\right), \quad C_{v}=\frac{3}{2}+\frac{N}{T^{2}}\left[\left\langle e^{2}\right\rangle-\langle e\rangle^{2}\right] \quad\left(\text { units } \epsilon=k_{\mathrm{B}}=1\right) .
$$




\section{Before swap}

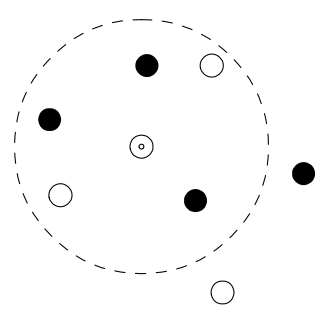

After swap

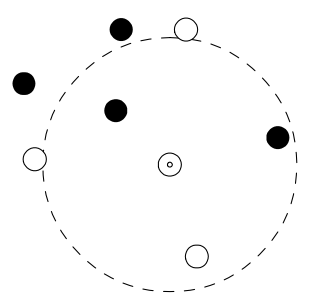

Figure 1: The local swap move. The A particles are depicted by full symbols, while B particles are open symbols. The (say) B particle picked randomly (signalled by a central mark) may be swapped with any one of the three A particles inside the sphere shown in the left part of the plot. After the swap (right), only two B particles could be exchanged with the picked B particle.

\section{The local swap Monte Carlo algorithm}

The Grigera-Parisi swap algorithm [9] consists in picking randomly a pair of particles of distinct type, $A$ and $B$, try to exchange their positions, and accepting this move with probability $\min \left\{1, \mathrm{e}^{-\Delta E / T}\right\}(\Delta E$ is the total potential energy change produced by the swap). If combined with standard MC, it is very effective in reducing the equilibration time. Nevertheless, there is a caveat: the acceptance of the swap move is very small, and it significantly decreases when the number of particles increases. Indeed, the closer the swaped particles are, the larger the swap acceptance becomes (this is a huge effect). With large systems, it is higly improbable to pick for the swap neighboring particles. To cure this problem, we have proposed the local swap.

In the local swap, the elementary MC step is either (with probability $p$ ) a single-particle displacement attempt or (with probability $1-p$ ) an attempt to swap particles. Therefore, for $p=1$ the algorithm reduces to standard MC. From here on we call local swap to the algorithm with $p=0.5$. The time unit $t_{0}$ is $N / p$ elementary steps. Our swap consists in picking a particle at random (the picked particle) and trying to interchange its position with that of a particle of opposite type (the swapped particle), chosen at random among those at distance smaller than $0.6 r_{c}$ (see Fig. 10. Yet, there is an intrinsic lack of symmetry. Indeed, let $N_{\text {old }}$ be the number of swappable particles around the picked particle in its original position, and $N_{\text {new }}$ the number of swappable particles for the picked particle in its final position. The probability of choosing the swaped particle is $1 / N_{\text {old }}$ in the original configuration, while it would be $1 / N_{\text {new }}$ in the final configuration. Detailed balance (see e.g. 2]) holds only if this asymmetry is incorporated in the probability of accepting the swap:

$$
p_{\text {accept swap }}=\min \left\{1, \frac{N_{\text {old }}}{N_{\text {new }}} \mathrm{e}^{-\Delta E / T}\right\} .
$$

The acceptance of the local swap is independent of the number of particles, and larger by a factor of 10 than for the original swap algorithm [9], already for $N=1024$. For the model $\sigma_{A} / \sigma_{B}=1.2$, the acceptance varies from $0.74 \%$ at $0.9 T_{\mathrm{mc}}$ up to $6 \%$ at $2 T_{\mathrm{mc}}$. For the $\sigma_{A} / \sigma_{B}=1.4$ is much smaller, actually of the order of $8 \times 10^{-6}$ at $T=0.83 T_{\mathrm{mc}}$, as could be guessed by the disparity in particle diameters.

The performance of the local swap below $T_{\mathrm{mc}}$ is far superior to the standard MC (Fig 21) and to the microcanonical method of Ref. [10] (Fig 3]). Furthermore, for both the $\sigma_{A} / \sigma_{B}=1.2$ and the $\sigma_{A} / \sigma_{B}=1.4$ models, local swap finds a crystallization phase transition, to highly disordered crystals on the bcc family, not reported on previous studies [11, 10]. 

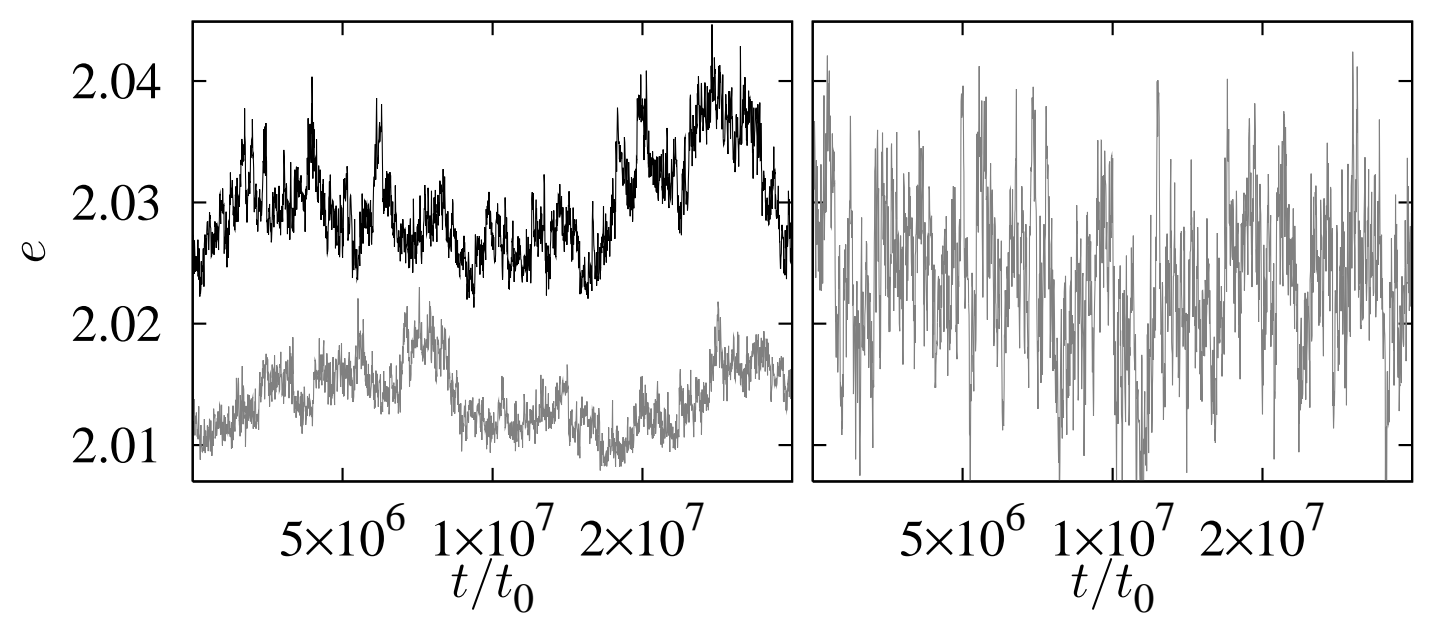

Figure 2: Comparison of the performance of the local swap (right) and standard Monte Carlo (left), as shown by the Monte Carlo history of the potential energy per particle (data points are the average of $10^{4} t_{0}$ succesive steps), for the $\sigma_{A} / \sigma_{B}=1.2$ model at $T=0.897 T_{\mathrm{mc}}$ and 1024 particles. The two standard Monte Carlo runs had as starting points thermalized configurations obtained with local swap. In our time window, the two standard simulations do not explore the same energy range. Instead the single local swap simulation explores the full energy range.

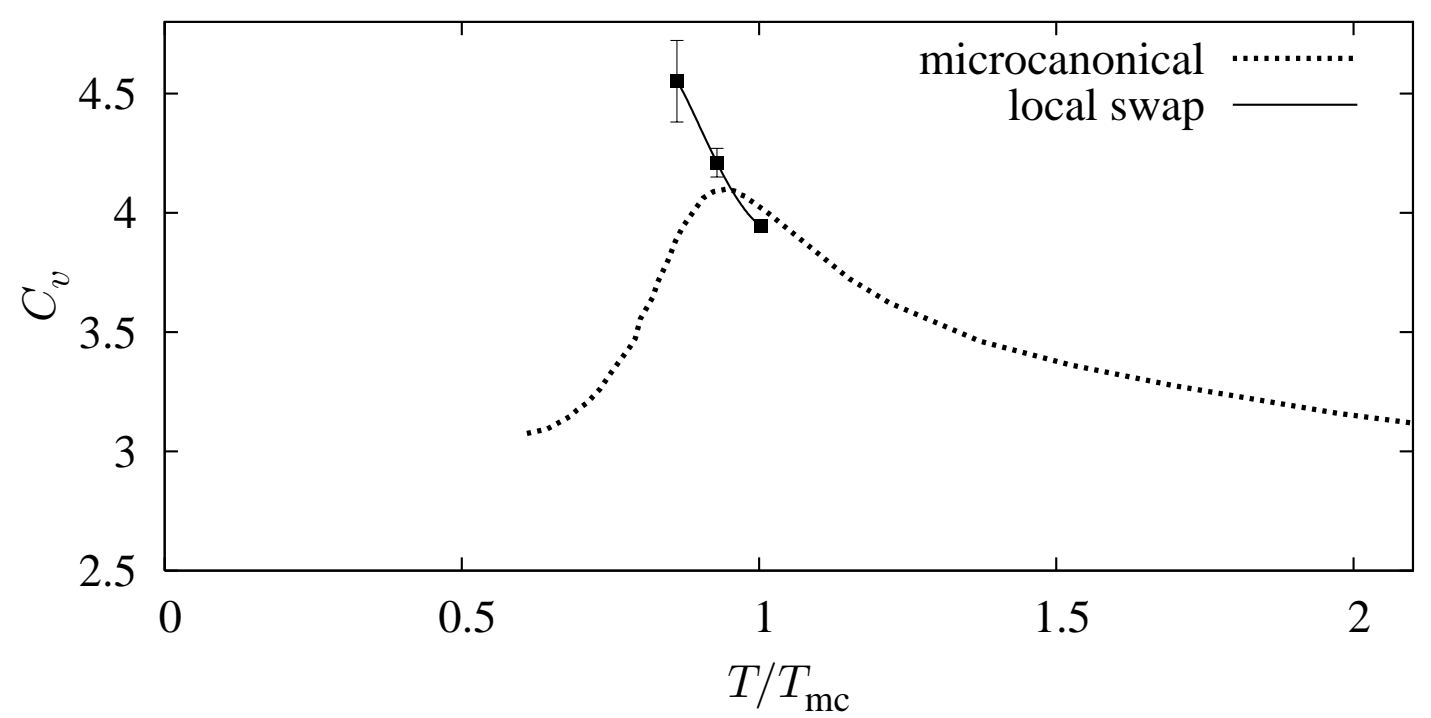

Figure 3: Specific heat of the $\sigma_{A} / \sigma_{B}=1.4$ model with 128 particles, as a function of temperature, as obtained with local swap $\left(10^{9} t_{0}\right.$ steps) and with the microcanonical method 10 (error estimates were not provided in Ref. [10]). Local swap produces a significantly larger estimate of the specific heat at our lowest simulated temperatures, signalling better sampling of configuration space and indicating that the microcanonical method is unable to thermalize at such low temperatures. 


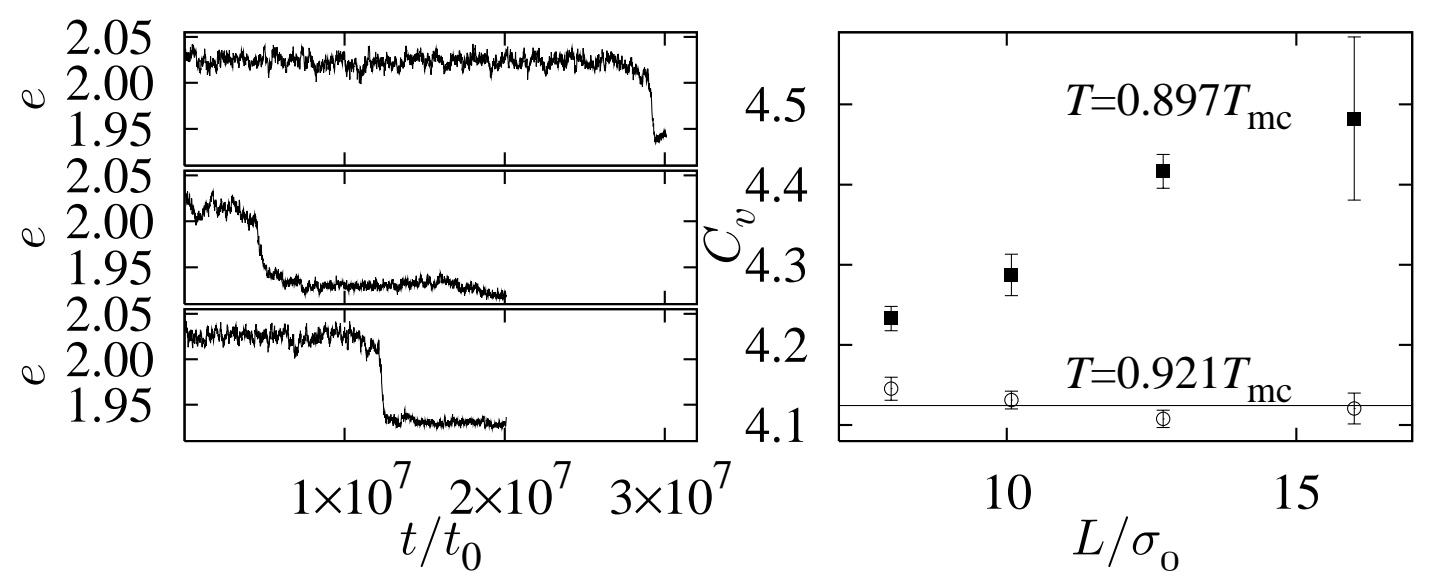

Figure 4: (Left) Examples from the 100 generated (local swap) Monte Carlo histories for the potential energy density of the $\sigma_{A} / \sigma_{B}=1.2$ model with 1024 particles at $T=0.897 T_{\text {mc }}$. One may clearly distinguish the metastable liquid from the crystallizing system, that starts as a sharp energy drop in all three runs. (Right) Specific heat for the model $\sigma_{A} / \sigma_{B}=1.2$ for sistems with 512, 1024,2048 and 4096 particles, at $T=0.921 T_{\mathrm{mc}}$ (empty symbols) and $T=0.897 T_{\mathrm{mc}}$ (full symbols), versus the size of the simulation box (the horizontal line is a fit of the $T=0.921 T_{\mathrm{mc}}$ data to a constant value, with $\chi^{2} /$ d.o.f. $\left.=4.89 / 3\right)$. At $T=0.897 T_{\mathrm{mc}}$, the specific heat increases with system size, while the energy density (not shown), is independent of the number of particles, within our accuracy.

\section{Finite size effects}

Even if not previously known, the liquid state is metastable in our soft-sphere models. Thus, the question arises of how to study the thermodynamic properties of a metastable phase. The underlying assumption is that the equilibration time for the metastable liquid phase is much smaller than the crystallization time. Our strategy has been to run several MC runs (up to 400 at the lowest temperatures [5]). On each run, the equilibrated metastable liquid is neatly separated from the crystallizing system (see Fig. 4. left). In the analysis we only consider histories whose metastable liquid part was selfconsistenly found to be longer than 100 exponential autocorrelation times (to avoid bias, we also discarded some 20 autocorrelation times before crystallization). Note that the central MC history in Fig. 4left does not meet this criterium (at this low temperature the autocorrelation time was $10^{5} t_{0}$ ). Then, one uses Eq.(1), with the metastable liquid part of the acceptable histories to obtain the results in Figure 4right (data from Ref. [5]).

\section{Conclusions}

We have given the first full description of the local swap algorithm [5]. We compare the performance of the local swap dynamics with the standard MC and with the microcanonical algorithm [10], concluding that local swap yield equilibrium data at temperatures where the microcanonical algorithm no longer thermalizes. Furthermore, local swaps finds crystal states not reported in previous work [10. We have shown how to estimate the specific heat in a metastable liquid state, finding at low temperatures tiny but clearly measurable finite-size effects [5]. 


\section{Acknowledgments}

We thank G. Biroli, G. Parisi and R. de Nalda for discussions. We were partly supported by BSCH-

UCM and by MEC (Spain), through contracts BFM2003-08532, FIS2004-05073 and FPA2004-02602. Simulations were carried out at BIFI.

\section{References}

[1] Debenedetti, P. G. (1997) Metastable Liquids, Princeton University Press, .

[2] Amit, D. and Martín-Mayor, V. (2005) Field Theory, the Renormalization Group and Critical Phenomena, World Scientific Singapore, in press.

[3] Götze, W. and Sjögren, L. (1992) Rep. Prog. Phys. 55, 241-336.

[4] Biroli, G. and Bouchaud, J.-P. (2004) Europhys. Lett. 67, 21-27.

[5] Fernandez, L. A., Martin-Mayor, V., and Verrocchio, P. (2006) Phys. Rev. E 73, 030501.

[6] Alcoutlabi, M. and McKenna, G. B. (2005) J. Phys.: Condens. Matter 17, 461-524.

[7] Alba-Simionesco, C., Coasne, B., Dosseh, G., Dudziak, G., Gubbins, K., Radhakrishnan, R., and Sliwinska-Bartkowiak, M. (2006) J. Phys.: Condens. Matter 18, 15-68.

[8] Morineau, D., Xia, Y., and Alba-Simionesco, C. (2002) J. Chem. Phys. 117, 8966-8972.

[9] Grigera, T. S. and Parisi, G. (2001) Phys. Rev. E 63, 045102-1-045102-4.

[10] Yan, Q., Jain, T., and dePablo, J. (2004) Phys. Rev. Lett. 92, 235701.

[11] Yu, C. C. and Carruzzo, H. M. (2004) Phys. Rev. E 69, 051201-1-051201-10. 